Association for Information Systems AIS Electronic Library (AISeL)

PACIS 2004 Proceedings

Pacific Asia Conference on Information Systems

(PACIS)

December 2004

\title{
An Empirical Study of Web Browsing Behaviour: Towards an Effective Website Design
}

Kwok-Kee Wei

City University of Hong Kong

Gek-Woo Tan

National University of Singapore

Hui-Kun Neo

National University of Singapore

Follow this and additional works at: http://aisel.aisnet.org/pacis2004

\section{Recommended Citation}

Wei, Kwok-Kee; Tan, Gek-Woo; and Neo, Hui-Kun, "An Empirical Study of Web Browsing Behaviour: Towards an Effective Website Design" (2004). PACIS 2004 Proceedings. 33.

http://aisel.aisnet.org/pacis2004/33

This material is brought to you by the Pacific Asia Conference on Information Systems (PACIS) at AIS Electronic Library (AISeL). It has been accepted for inclusion in PACIS 2004 Proceedings by an authorized administrator of AIS Electronic Library (AISeL). For more information, please contact elibrary@aisnet.org. 


\title{
An Empirical Study of Web Browsing Behaviour: Towards an Effective Website Design
}

\author{
Kwok Kee WEI \\ Department of Information Systems \\ City University of Hong Kong \\ isweikk@cityu.edu.hk
}

\author{
Gek Woo TAN and Hui Kun NEO \\ Department of Information Systems \\ National University of Singapore \\ tangw@comp.nus.edu.sg \\ neohuiku@comp.nus.edu.sg
}

\begin{abstract}
Prior studies have suggested that a good website design which facilitates a person's web browsing behaviour would generally lead to better user performance. In this research, we examine user website behaviour as a way to understand Website design. Main theoretical contributions of this research are the illustration of the flow of cognitive processes during the website browsing and the establishment of various website design dimensions in relation to user performance.
\end{abstract}

Keywords: Website design, user performance, cognitive mapping

\section{Introduction}

Badly designed Websites frustrate users and cause them to leave as they cannot find what they need (NUA, 2002). The core question lies in how Websites should be designed to facilitate users in finding what they need and increase satisfaction while accomplishing their tasks (Abdelmessih et al., 2001).

Wayfinding theory (Passini, 1984) postulates that a person would perform cognitive mapping, use it to generate a series of plan in order to navigate the real environment and complete his task. Morville (2002) highlighted that wayfinding theory is applicable in the Website environment as it is about "designing systems that help people find what they need". Furthermore, due to the dynamic characteristic of the Website environment, it is imperative for Website environment to facilitate browsing (i.e. wayfinding) than the real environment. Moreover, as Websites expand in size and complexity, they have to be designed to reduce the user's browsing efforts in completing his task (Modjeska, 1997).

Therefore, the goal of this research is to build a conceptual model of an effective Website design that aimed to reduce cognitive overhead involved and facilitate website browsing in its optimal manner. The contribution of this research is the establishment of Website design dimensions, which influences browsing behaviour, as well as formation of guidelines to assist designers to better identify areas for improvement and create effective Website.

\section{Literature Review}

Wayfinding is the cognitive process in which a user adopts to find his way to destination(s). It made up of three processes: cognitive mapping, decision generation, and decision execution (Passini, 1984). 


\subsection{Cognitive Mapping}

Cognitive mapping ${ }^{1}$ is processing the information from the environment in conjunction with the past knowledge of a person.

Passini (1984) named all the necessary information needed as environmental information and categorized it as sensory (Is), inferential ( $I i)$ and memory $(I m)$ information. The sensory information is directly related to the current environment that a person is in, while the other two are indirectly related as they are information drawn from him. The sensory information (Is) is made up of architectural and spatial characteristics of a setting, signs, and people who provide cues to a person. Inferential information (Ii) is what he infers from the current environment, like the "Exit" sign above a door implies that it leads to the outside of a building. Lastly, memory information (Im) refers to his past knowledge or cognitive map.

The output of cognitive mapping is a person's updated cognitive map, a mental representation of the real environment at a particular moment in time, and is made up of three components: landmark, route, and survey knowledge (Thorndyke and Hayes-Roth, 1982). Landmark knowledge tells what something is and they can be points of identity, location, navigation aid, and orientation (Golledge, 1999). Route knowledge is a representation of the paths connecting the various landmarks, informing a person where something is (Downs and Stea, 1977). Lastly, survey knowledge is the topology of the environment or good understanding of the surrounding area that allows a person to get to a destination efficiently.

\subsection{Decision Generation}

After cognitive mapping, the next process is decision generation in which a person generates and structures a series of decisions that would complete his task. All these decisions have 2 aspects planned behaviour and expected image. Planned behaviour is what a person has planned to execute, while expected image is what he perceives of the outcome of the execution. This would then lead to decision execution.

\subsection{Decision Execution}

During decision execution, the decisions are transformed into actions by comparing predictions about the environmental features and the information obtained from the current environment, i.e. comparing the expected image of a decision and perceived image of the current environment, which is also Is. If the expected image matches the perceived image, then a person would execute his decision which is a behavioural action. If they do not match, this implies that certain information is lacking from the environment in order to act out his decision. As such, the decision is not executed and becomes a new subtask. This prompts a loop-back to the cognitive mapping process and restarts the whole procedure.

In fact, the environment and task will dynamically change and the knowledge of the person will dynamically update as he moves through the environment. Similarly, the cognitive maps would be updated whenever information is added or forgotten. If the cognitive maps have changed, the plans would change as well.

\footnotetext{
${ }^{1}$ Downs and Stea (1977) defined cognitive mapping as "an abstraction concerning those cognitive or mental abilities that enable us to collect, organize, store, recall and manipulate information about the spatial environment".
} 


\section{Research Methodology}

This study focuses on the notion that an effective Website design permits a user to navigate easily and accomplish his task effectively. Wayfinding theory is used to understand user behaviour for it also encompasses many issues like interaction design, usability engineering, information architecture, knowledge and content management (Morville, 2002).

\subsection{Case Background}

The Website used was Dell's (http://www.dell.com, accessed: Oct 2002). Dell's Website made "computer buying painless", and thus, it is rated as one of the top 10 classic Websites this year by PC Magazine (Rubenking, 2003). Other comments from their customers surveyed by BizRate.com on Dell were very positive, "this was the best shopping and buying experience I've ever had!", "my second experience purchasing ... ... once again a pleasure", "very easy and user friendly to place the order".

\subsection{Subjects}

Six subjects were recruited using an attractive high fixed payout. Their demographics were three males and three females, average age was 22 and they seemed to be quite comfortable and skilful with the Internet. This group of subjects also represented the major group of the Internet population who was also responsible for a large portion of the online sales last year (Greenspan, 2003).

\subsection{Data Collection}

The assigned task was for the subject to purchase either a desktop or notebook computer of his/her choice from Dell's Website. The first step was for the subjects to fill up a pre-interview survey. The experience of the subjects and their initial impressions of the investigated Websites were obtained from the pre-interview surveys. They next proceeded to do the assigned task. Along the process, the subjects would need to think aloud their thoughts and actions by stating what they see, their location, how they got there, whether they could recognize the steps taken etc. In addition, a special software program was used to record all actions on the monitor screen as well as voices of the subjects and interviewer. After completion of the assigned task, the subjects filled up a post-interview survey regarding their experience at the Website. Lastly, a short open-ended interview was conducted, summarizing their thoughts on the interview session which was taped using a video camera.

\subsection{Data Measurements}

The three processes of wayfinding are difficult to measure directly as they are internal to the person, hence only indirect measurements were used. For cognitive mapping, much literature had dealt with measuring it through drawings of the real environment (MacKay et al, 1975; Lynch, 1960). It tests the ability of the person in organizing and processing the information. Decision generation was measured through a diagram of hierarchy of decisions as they are self-generated by a person on the subconscious level which may go unnoticed by him, using the protocol developed by Passini (1984). Decision sequence diagrams and structure diagrams were attained by transcribing the coding of the observations. Sequence diagrams code the chronological order in which environmental information are extracted and decisions are generated. Structure diagram organizes the hierarchy of tasks and subtasks and the points of decision execution. Lastly, for decision execution, it was measured through the action taken as the result of it would signify whether the task was accomplished, which was then sent as a feedback to the person, as well as the satisfaction gained in accomplishing it. 


\section{Research Findings \\ 4.1 Cognitive mapping}

It was observed that the subjects were constantly extracting environmental information and it could be similarly broadly classified as sensory, inferential, and memory information. Most of the time, it was noticed that the subjects were taking in sensory information, for example in Dell's Website, "I see three types of Dimension desktop", "Select country option...". Thus from our observation of the subjects, the sensory information was mainly comprised of spatial characteristics and signing of the Websites, analogous to the frames, window screen, tables, hyperlinks, navigation bar, sitemap etc. The third type of sensory information would translate to the online assistant, FAQ, and alternate hyperlink description. However, this third type was not widely observed during subjects' exploration, only 1 subject turned to the FAQ in the hope of finding "the warranty of (that) computer".

The second most documented was inferential information, while the least was memory information. Inferential information was comments by subjects based on the current Website environment, like on Dell's Website, "this is a simple and nice homepage design", "this Dimension desktop model is cheaper than Dimension 8200”. As for memory information, the subjects seldom recalled information from their memory. When they did recall, it was regarding how similar Dell's homepage looked like "other commercial Websites", and desktop "looks familiar”.

Like in the real environment, the subjects were observed to possess previous knowledge or cognitive map of the Website, and after their exploration, they had developed a new or updated ones. This was observed from the drawings done by the subjects of the Website. The drawings were noted for features or items that could translate to mental representations in the subjects' cognitive map.

With regards to the cognitive map, Farris et al (2001), Kim and Hirtle (1995) argued that Webpages could be seen as landmarks and hyperlinks as routes, while survey knowledge was analogous to the layout of landmarks (Webpages) and paths (hyperlinks). Golledge (1999) suggested an alternative view of landmarks - components or features within a Webpage, such as the title header, navigation bar, company logo, and main text body. As for survey knowledge, the layout of landmarks could be on the Website level, a sitemap, and on the Webpage level, placement of features. Hence the accuracy of the drawings was measured on these levels, i.e. the identification of the exact main features and its placements, as well as an approximate of the depth and breadth of the Website structure.

The analysis of the drawings showed that the subjects' initial cognitive maps of Dell's Website matched closely with the actual one. Majority of the subjects had visited Websites that sold computer hardware, all of them had heard of Dell and only one had actually visited Dell's Website before. The subjects had managed to visualize correctly majority of the landmarks and their placements for Dell's homepage, like the company logo, navigation bar, product catalogue, and content area. They could also visualize that Dell's Website has a deep structure. The high similarity in the drawings of both cognitive map and actual Website suggests that the user adopts mental images of the Website based on past experiences. 


\subsection{Decision Generation}

The subjects' decision generation process was observed through the sequence diagrams. Subjects would pause to think before making the decision through comments like "let me see...", "how do I do this...", "where is the catalogue now...". During these pauses, subjects were actually looking at the Websites, attempting to organize the information that they had and generating the possible decisions to accomplish a task based on his cognitive map. The structure diagrams traced the actual decision generation. Faced with the assigned task of purchasing a computer online, the subjects broke it down to 2 subtasks - browsing for a suitable product to purchase and looking at the ordering information. These subtasks were then further broken down to browsing the current promotions, looking at the product catalogue, comparing prices and specifications of the products etc (see Figure 1).

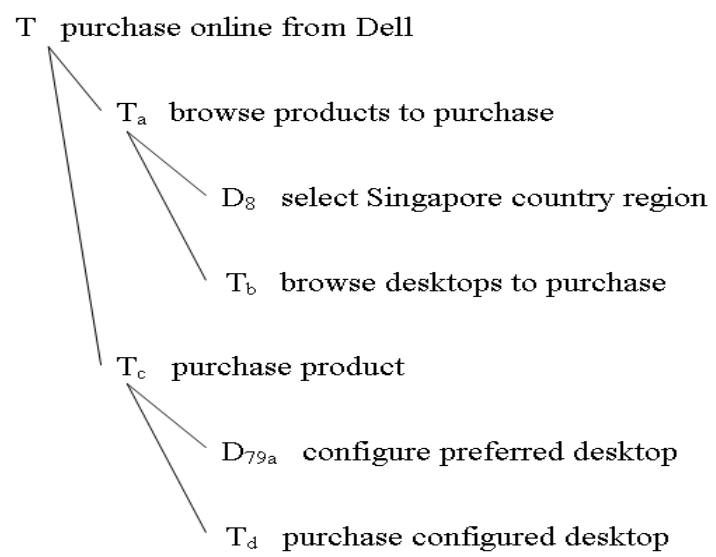

Figure 1. Subject B's decision hierarchy in Dell's Website.

\subsection{Decision Execution}

This last process is also similar in both real and virtual Website environments in terms of the decisions that are executed and unexecuted. From the sequence diagrams, it could be observed that the confirmation of a decision being executed was the revealing of another Webpage based on the hyperlinks clicked and selections made, or changes in price of system according to changes in configuration of system etc (see Figure 2). As for unexecuted decisions, they became new subtasks, looping back to cognitive mapping and restarting the whole procedure, illustrated from the example of the subjects figuring out how to compare different computer models in Dell's Website (see Figure 3).

\subsection{User Performance}

All subjects had managed to select a computer system that they preferred and "purchased" it. On the whole, the Website was given a rating of 6 out of 7 by the subjects for being able to assist them in accomplishing any tasks effectively. In terms of efficiency, subjects spent about an average of 46mins in Dell's Website, selecting and comparing the products to purchase. The Website was given a rating of 6 out of 7 by the subjects for being able to assist them in accomplishing any tasks efficiently. The high degree of the design dimensions found in the Website helped to notify the subjects where the information was. Hence, subjects felt that they could find what they needed within a few minutes or clicks. 


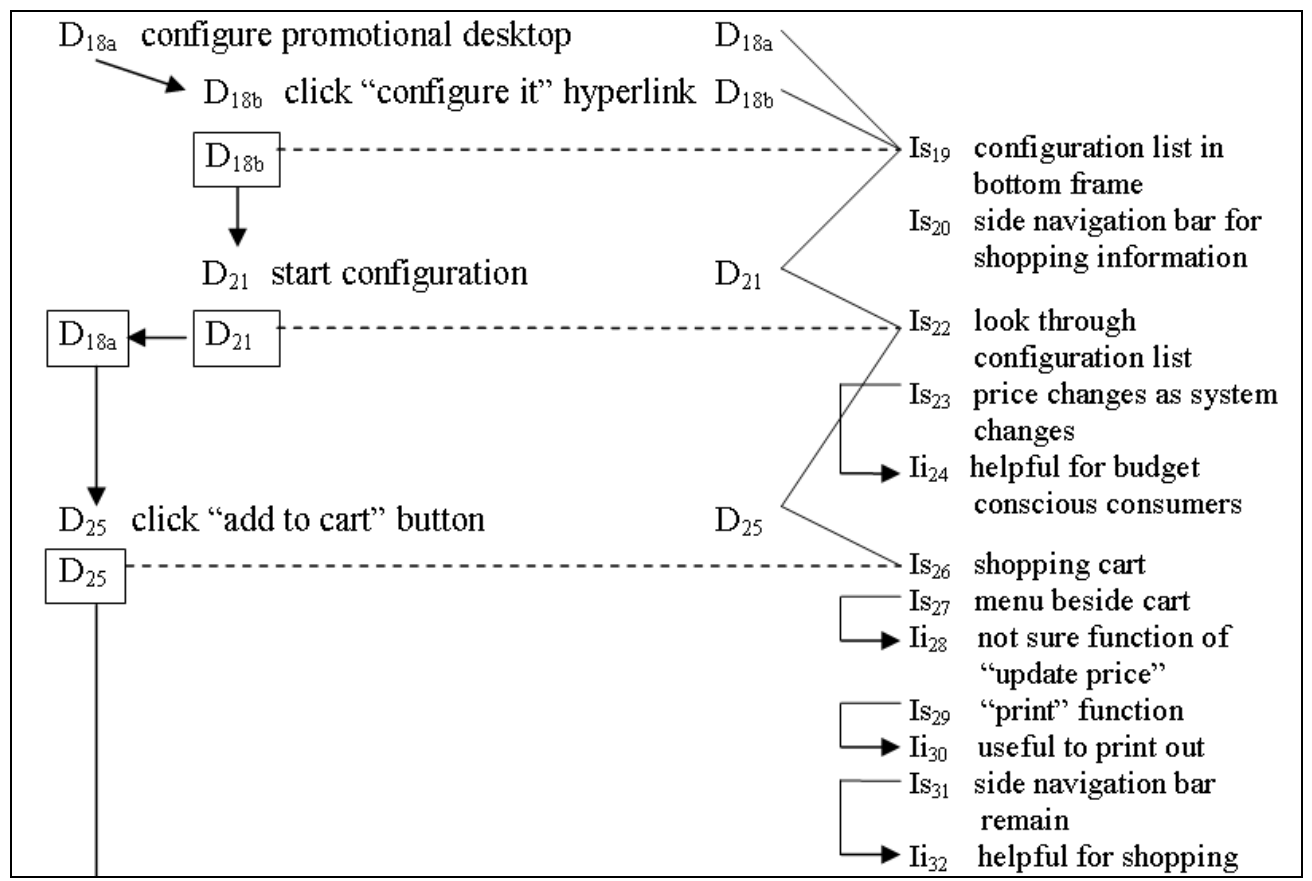

Figure 2: Price changed while Subject F configured Dell's product.

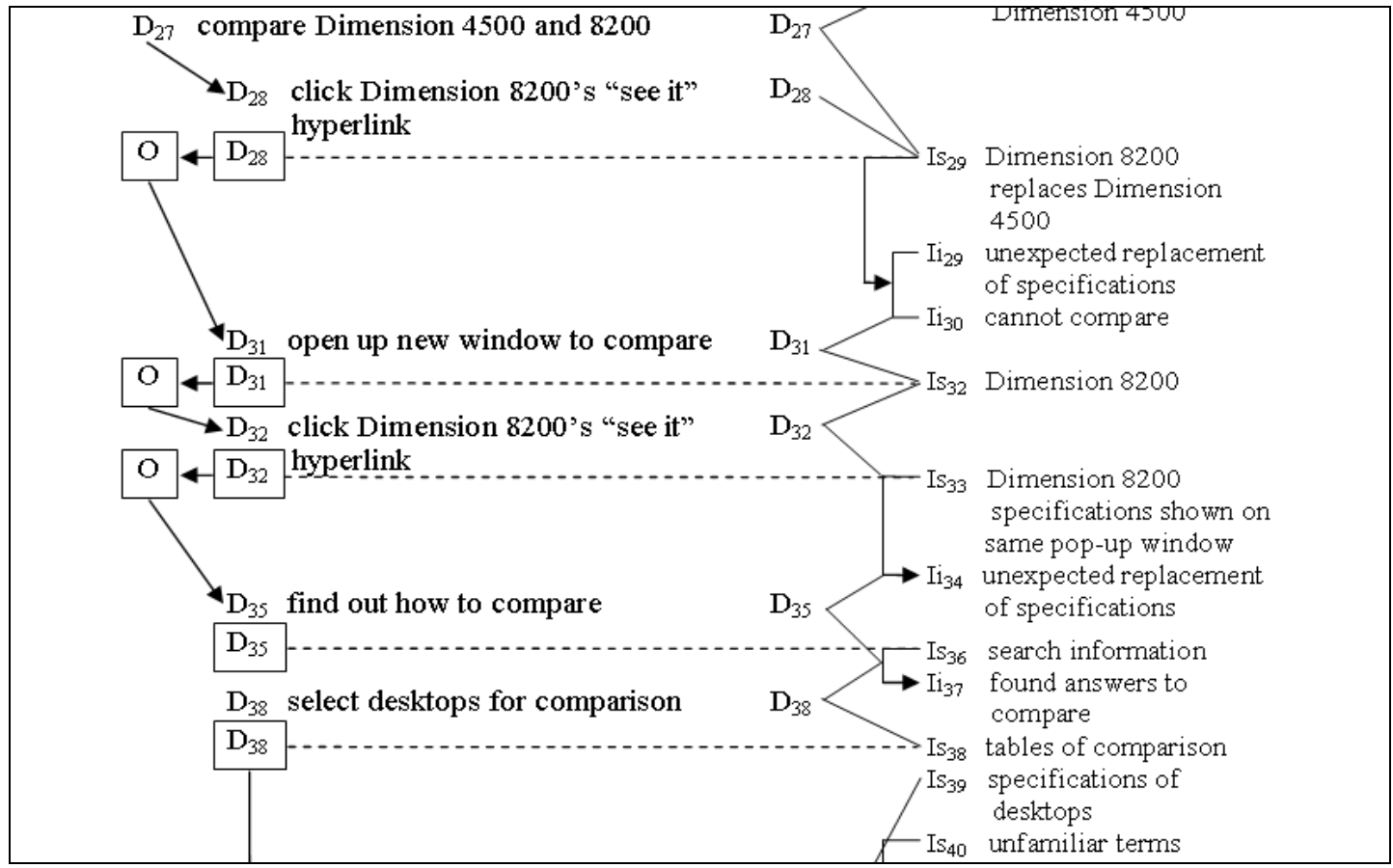

Figure 3: Subject C used different windows to compare Dell's Dimension products

In measuring the subjects' satisfaction, there were a few "lostness" registered. "Lostness" was felt as the subjects thought that the categorization of product type and usage purpose was confusing when it crossed together, and had experienced difficulty getting to the "mainpage", as well as "how do I compare these models here?", "I can't seem to find the (computer) warranty". Fortunately, it was considered to be minimal and of little effect on the overall satisfaction as the 
subjects managed to find what they needed after a few trials and errors. Thus, they gave a high rating on satisfaction to the website, and on average rating of 5 out of 7, they felt that the visit was interesting and enjoyable.

Besides that, the Website had earned high satisfaction rating and good comments as the subjects considered that it had an "attractive homepage, like the personalization touch" with the selection of country region, and was "very professional looking". An average of 3 counts per subject was noted on these good comments. As a result of the effective Website design and good user performance, the subjects expressed their "trust" for Dell and intentions to purchase, or recommend to others were also high.

The results of the study showed that an effective Website is an environment that facilitates wayfinding and generates high user performance.

\section{Discussion}

The subjects ranked Dell's Website highly on informed purchase decision, consistency with the general Web conventions, presentation style, and navigation style. They commented that it had "relevant and updated information", was "neat, clear, clean, consistent and easy to read", and had "proper menus to guide (them)". We present the discussion along the three dimensions content, presentation and navigation, there emerged from the data analysis

\subsection{The meaning conveyed or implied by the Website content}

As most users are surfing on the Internet to gain specific information, it is imperative to understand the key characteristics of information quality from the user's perspective (Aladwani and Palvia, 2002). Katerattanakul and Siau (1999) measured the quality of information content and found that context is a major factor towards the user's comprehension of the Website. Johnson-Laird (1983) noted that providing relevant information is important as it assists in building a user's cognitive map by mapping it onto existing or new one.

A subject remarked that the Website, the "information was given in a very user friendly manner". Overall, with such good quality of information, subjects were able to construct good description regarding the different Webpage landmarks, making each Webpage unique. As such, subjects could form the landmarks in their cognitive maps quickly and generate decisions quickly to identify which landmark to focus on and know exactly where to find it (see Figure 4).

Furthermore, the contents found in this website helped the subjects to form well-informed decisions during decision generation in selecting a product to purchase, and helped them to assess their decisions for execution. It made the subjects felt confident that they were looking at the information exactly or close to what they need, "I think I can find the product warranty in this table", "the product I want should be in here". With such confidence, they did not need to analyse whether the information is accurate, updated etc, "I trust Dell to put up the latest products and information".

Focusing on comprehensibility of information, Dell's Website had mid degree as multiple computer jargons were used in the description of their products. Fortunately, Dell's Website had short description and footnotes accompanying the terms such that the subjects could understand the product specifications easier and place them in their cognitive maps so as to compare them 
mentally, as a subject commented, "I like the description, very clear and I can understand them quite well".

As for customization of information, Dell's Website had subsites representing different countries. Such customization not only created home-like environments for the subjects, "feel very patriotic looking at (my country) flag", it also helped to narrow down the information that they had to go through, making it more convenient for them to accomplish their tasks.

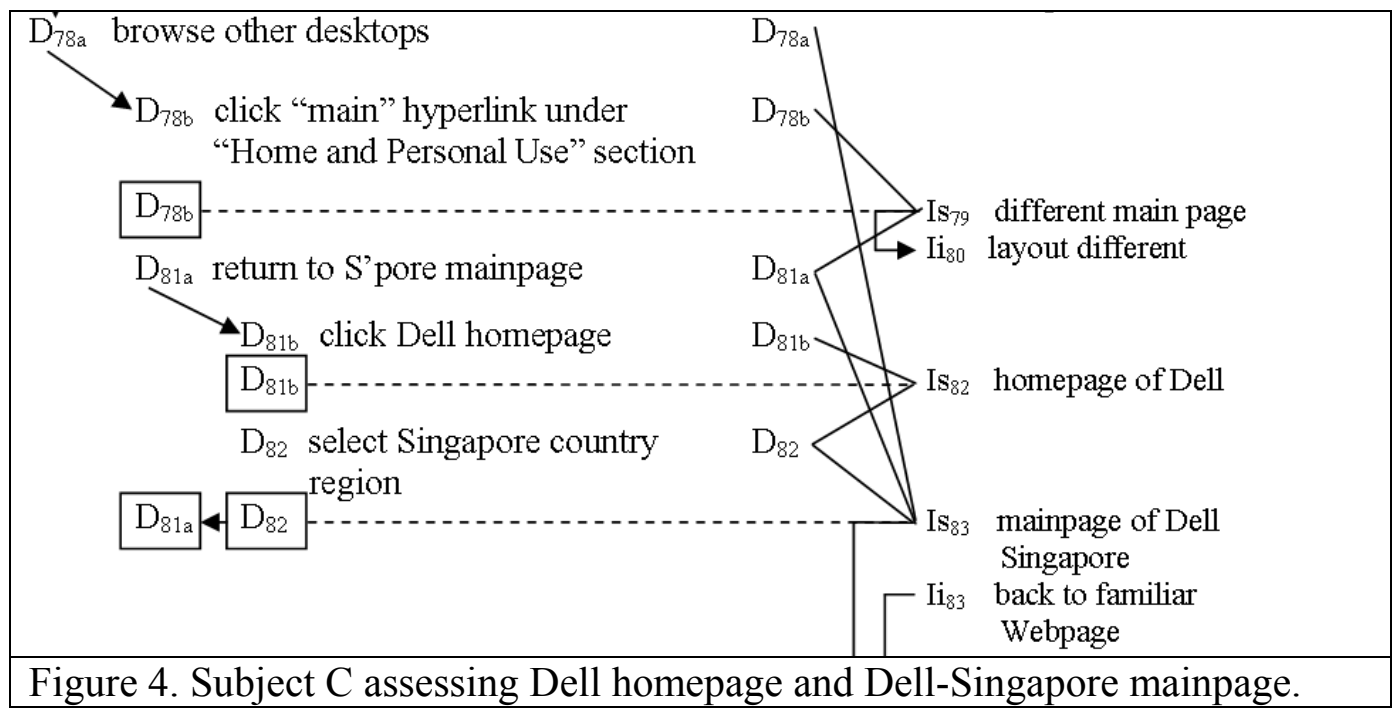

\subsection{The appearance or outward forms of the Website}

Graphical presentation, like icons, colours, images and animations, are used to present the Website more vividly and to increase satisfaction (Zhang et al., 1999). A well-presented Website would also improve Website navigation (Zhang et al, 2000). In essence, any Webpage should convey coherence, possessing a consistent presentation style that is used for all Webpages within the Website. In our study, the appearance of Website is instrumental in facilitating browsing to form better landmark and route knowledge, reduce cognitive load on subjects, and improve perception of information in order for subjects to perform better cognitive mapping and assessment of decisions for execution.

In particular, the consistency of design and compliance with the general Web conventions helped to ease the subjects' learning process in navigating the Website. First, the consistency of design helped to create a sense of identity and thus, "I can recognise that I'm still in Dell because Dell's logo and everything look the same to me". In addition, the consistent placement of Dell's logo, navigation bar etc, allowed the subjects to form distinct landmarks such that they could easily form a cognitive map of how the next Webpage should look like and immediately focus onto a particular area, "I'm focusing on this area because I know that the description is in here". Hence, consistency of design for Dell's Website facilitated the subjects' browsing behaviour.

Second, Dell's compliance with the general Web conventions facilitated the cognitive mapping of the Website. The subjects, having been exposed to many Websites, were accustomed to some Web conventions, "Dell definitely follows the normal Web conventions", "everything looks pretty much the same as in any commercial Websites". For example, Nielsen and Tahir (2002) 
found that most Websites placed the navigation bar on top and the company logo in the top left corner, which was the exact positions found in Dell's Website. Thus, there was less cognitive load on the subjects as they could easily learn the design of the Website based on past experiences. A few subjects had noted that they "know where to go" without wasting their "time trying to figure (their) way around the Website" as the presentation of the information in the Dell's Website was “more predictable”.

The good graphical design also facilitated browsing by making it easier for the subjects to read information without boring them. The appropriate inclusion of graphics would capture the subjects' attention better than a plain text Website. As the saying goes, "a picture is worth a thousand words", well-represented graphics of landmarks could replace any text description of the landmarks (Fiore et al., 2003). Hence, the subjects could form better landmarks by using the graphics displayed on Dell's Website.

Next was the typography of Dell's Website, the black text over white background gave the maximum colour contrast (highly recommended by Nielsen and Tahir (2002)) and made it easier for the subjects to read, "this is quite easy to read", "I'm glad I don't have to highlight the text to read it". With high legibility, the subjects would then be able to extract even more information to form a proper cognitive map as well as to assess decisions to execute. Lastly, for Webpage layout, the subjects did not need to scroll much to view the information, and thus, they were able to perceive all the information on the Webpage at a go and concentrate on it.

\subsection{Navigational landmarks of the Website}

A well-structured Website has a hierarchy of Webpages should be built "that feels natural and well-structured to the user, and does not interfere with their use of the web site or mislead them" (Lynch, 1995). A user should have a good overview of the Website to reduce the cognitive overhead in establishing his cognitive map. He would then be able to understand and recognize his current Website location, and navigate easily (Larson and Czerwinski, 1998). In navigating Dell's Website, the subjects did not find it difficult as there were several navigation aids that allowed them to easily search for information. In fact, the subjects were using only the navigation bars and category hyperlinks, but not the search engine. Thus, those navigational aids formed navigational landmarks in the subjects' cognitive maps and they were used like marked trails to navigate the Website.

The high degree of orientation cues found also helped to facilitate browsing. The subjects felt that Dell's Website had a "predictable (Website) structure" and clearly labelled hyperlinks, and they were confident that the "(hyper) links link to the pages (they) expect". Subjects also noted that even first-time visitors or "novices will find it easy to navigate" as they did not need to interpret the meaning of the cues. As Krug (2002) noted, the less that the users have to think about navigation, the better the navigation system is.

The well-labelled hyperlinks were important as subjects could better orientate themselves and predict the content underlying it. In the decision execution process, the subjects were assessing the words used in the label and attempting to match it with their cognitive maps. Hence, they could identify which hyperlink to click and the title header of a Webpage would assure them that they were on the right track. Thus, Dell's Website was quite "predictable". 
The last orientation cue found was the sitemap, but none of the subjects used it. Only one subject clicked it to see the organization of Dell's Website, "I like to check out the sitemap and it will tell me the organization of the Website." The sitemap represents a high-level overview of the Website and it would help to form the survey knowledge in the users' cognitive maps. As the subjects were mostly relying on the navigation bars and category hyperlinks, i.e. landmark and route knowledge, they did not need to form the full survey knowledge of the Website to navigate.

Judging from the subjects' drawings, they had some initial survey knowledge regarding the organization of Dell's Website (see Figure 5). This implied that the organization matched with the initial cognitive maps of the subjects. Hence, it facilitated the subjects' browsing as fewer changes were made to their cognitive maps.

\subsection{The flow diagram of the browsing behaviour}

This study focuses on how to enhance user performance through better Website design. Drawing from prior studies, we conducted an empirical study to examine web browsing behaviour. From prior literature and results of this study, a new flow diagram of the browsing behaviour was formed (see Figure 6). This new diagram illustrates the relationships between the processes and the components of web browsing. Since Web browsing is an iteration process, hence there are links leading from decision execution and behavioural action back to the top and restart the entire process. In the diagram, a big rectangular box, encompassing the browsing task, user, and the Website environment, is to signify that the 3 components dynamically changed or updated as the user progresses to complete his browsing task in the website environment.

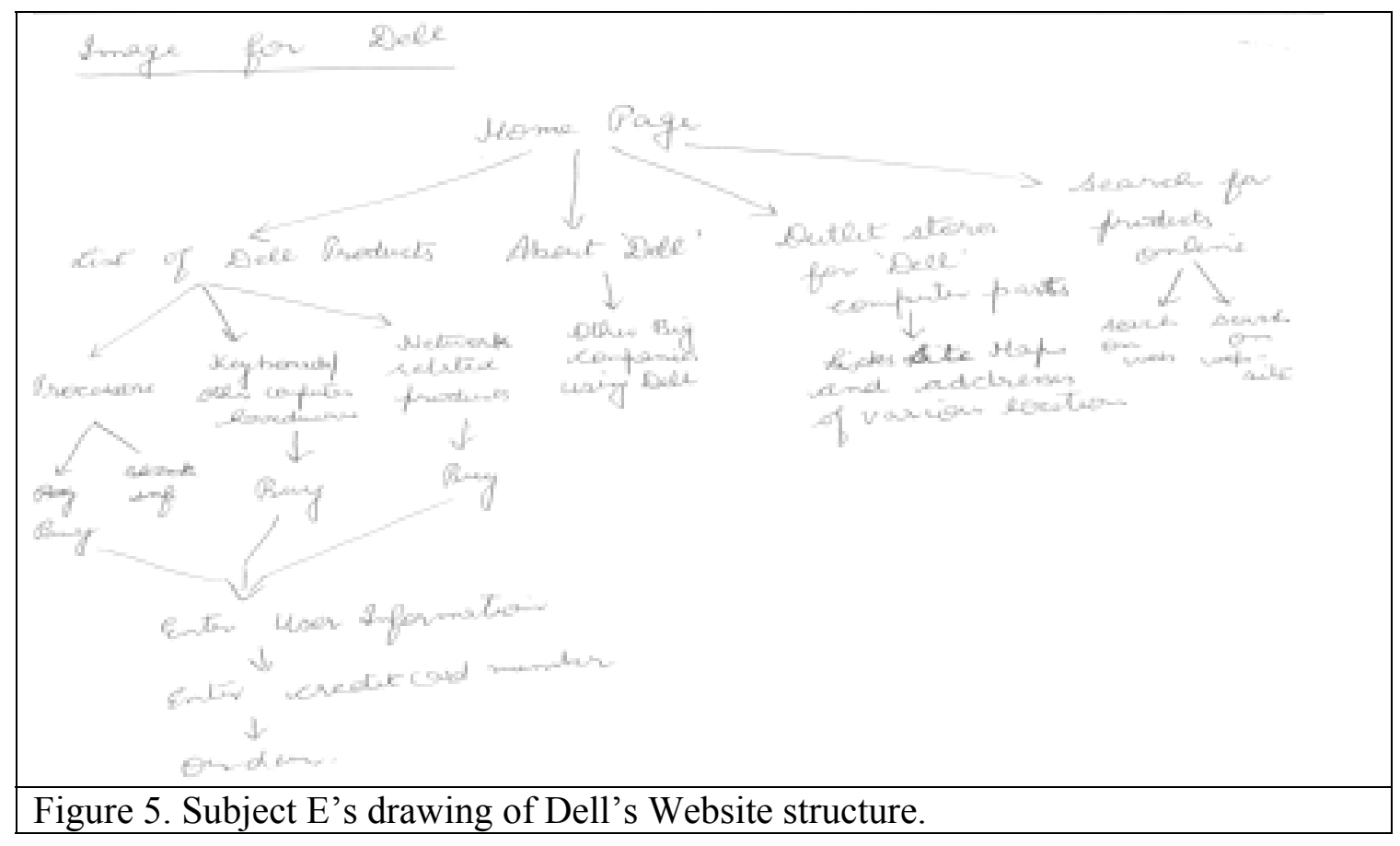




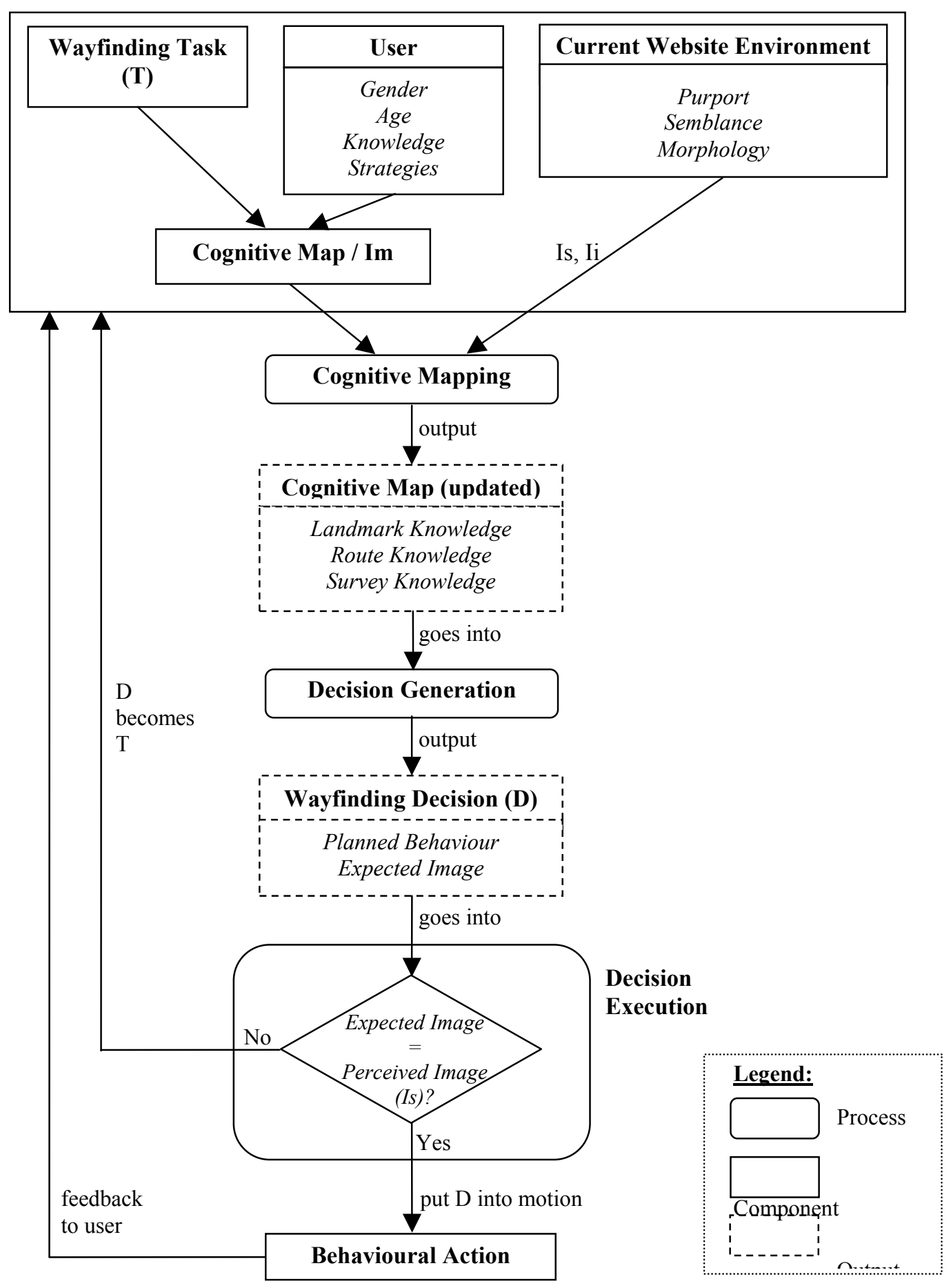

Figure 6. New wayfinding diagram. 


\section{Conclusions}

\subsection{Implications to Academics}

The benefit of incorporating user behaviour into Website design is that it could lead to better human-computer interaction. This would require an extensive research ranging from the importance of user's memory on navigation to the visual effects of the Website features on the user's perception, revealing interesting results of user behaviour. With regards to the user's memory, it plays an important role in the user's familiarisation of the real environment as places and signs remain unchanged for a period of time. However, in the Website environment where Websites change frequently, a user is constantly faced with new designs of a Website that it may be pointless for a user to remember what information is located where within the Website. Hence, it would be interesting to know how important a user's memory is in navigating the Website.

Moreover, more research on the cognitive mapping process and its product would be beneficial. There is still a gap between what the user expects to see and what he actually sees in the Website. Currently, it is the user who closes the gap through learning to map the actual Website to his mental model and formulate his browsing strategy. By understanding cognitive mapping, an effective Website design can be formed which shortens the user's learning curve and supports multiple browsing strategies.

In addition, academics can also investigate whether the Website features would actually influence a user's perceived trust of the Website and his behavioural intention, as there were subjects' comments on Dell's Website such as "I trust Dell", "Dell is stable and professional". However, trust and behavioural intention are dependent on lots of other factors like company policies, security etc. Hence this is a possible extension of the research as it would be of value to better understand the design of commercial Websites.

\subsection{Implications to Practitioners}

Results of this research showed that practitioners should focus on the users' needs, which is to find their way around in the Websites easily, comfortably, and enjoy the process. Practitioners sometimes redesign their Websites for a variety of reasons - improving the Webpage layout and graphics, or giving a fresh look to attract repeat visitors. They should take note that the new design should still enable repeat visitors to comfortably navigate around and locate what they need. Also, Website design should be clear in its purpose (for commercial, recreation, or personal), to the types of users who would possibly visit the Websites (first-time users versus experienced users), as these factors would affect how the users find their way around the Website. Hence, practitioners should re-prioritise Website features that facilitate wayfinding when designing Websites. For example, consistent presentational style and the presence of navigational aids and orientation cues are very important with regards to efficiency and satisfaction.

Practitioners can also draw from the browsing protocol and find out how effective their Websites are. It presents to them a systematic attempt to collect data on the direct experience of user and understand how certain Website features affect the user performance. With an effective Website that facilitates browsing, user would then be able to find what they want successfully. Thus, the usage of the browsing protocol would be appropriate for practitioners as well. 


\subsection{Limitations and Future Research}

For future phases of the research, an investigation can be conducted on the importance of a user's memory in navigating through a Website. As mentioned earlier, a person's memory is very important in helping a person to recall the path to a destination in the real environment. The cognitive mapping found in this study may not be appropriately generalized to those people who never surf the Internet and to those who have no previous experience with the kind of websites that the study examined. In such a case, memory information (Im) is absent. Also, the importance of a user's memory in navigating the virtual Website environment is questionable as the Websites change so frequently that little remains the same for a user to remember and recall. Hence, an exploration into the user's memory may reveal interesting results.

The value of the research is constrained by choosing the Dells' website and the six subjects who had quite some skills and experience with the Internet and online shopping. With a well designed website and the skilled subjects, the study may not find the true wayfinding process. Hence, studies could be conducted with respect to those websites with bad design and novice web users. In such, the researchers may or may not match the new wayfinding diagram in figure 6 .

From our study, it can be observed that at least three website design dimensions are important to the user performance. They are (1) the meaning conveyed or implied by the Website content; (2) the appearance or outward forms of the Website; and (3) the navigation landmarks of a Website. Some prior studies have shown that, other website design dimensions, such as security, playfulness, privacy, of the website are also important to user performance. Hence, experiments can be conducted to investigate the main and interactivity effects of these Website dimensions on user performance.

Furthermore, an investigation on the intervening variables, like cognitive map, strategies, and their effects on user performance can be conducted. The challenge lies in the measurement of the cognitive map as it is still in its early stages. Hence, this investigation would be of value to understand in-depth the user behaviour in the Website and the extent to which the influence of these intervening variables on browsing and user performance.

\section{References}

Abdelmessih, N., Silverstein, M., and Stanger, P. "Winning the Online Consumer: The Challenge of Raised Expectations," The Boston Consulting Group, 2001.

Aladwani, A.M., and Palvia, P.C. "Developing and validating an instrument for measuring userperceived web quality," Information \& Management, 39, 2002, pp. 467-476.

Campbell, C.S., and Maglio, P.P. "Facilitating navigation in information spaces: Road-signs on the World Wide Web," International Journal of Human-Computer Studies, 50, 1999, pp. 309-327.

Comrey, L. A First Course in Factor Analysis, Academic Press, New York, 1973.

Downs, R. M., and Stea, D. Maps in Minds: Reflections on Cognitive Mapping. New York: Harper \& Row, 1977.

Fortune. "Web Design - Design Matters," Fortune, 1 Dec 2000, URL: http://www.fortune.com/indexw.jhtml?channel=artcol.jhtml\&doc_id=00000071, accessed on $02 / 05 / 2003$. 
Gehrke, D., and Turban, E. "Determinants of Successful Website Design: Relative Importance and Recommendations for Effectiveness," Thirty-second Hawaii International Conference on System Sciences (HICSS), Maui, Hawaii, Jan 5-8, 1999.

Golledge, R.G. Human Wayfinding and Cognitive Maps. In: Golledge, R.G., Wayfinding Behavior - Cognitive Mapping and Other Spatial Processes. The Johns Hopkins University Press, 1999, page 5- 45.

Johnson-Laird, P.N. "Mental models," In Foundations of Cognitive Science, Posner, M.I., Ed. MIT Press, Cambridge, MA, 1989, pp. 469-499.

Kaplan, S. "Environmental Preference in a Knowledge-Seeking, Knowledge-Using Organism," In The Adapted Mind: Evolutionary Psychology and the Generation of Culture, Barkow et al., Ed. New York: Oxford University Press, 1992, pp.581-600.

Katerattanakul, P., and Siau, K. "Measuring Information Quality of Web Site: Development of Instrument," Twentieth International Conference on Information Systems (ICIS), Charlotte, North Carolina, Dec 12-15, 1999, pp. 279-285.

Larson, K., and Czerwinski, M. "Web Page Design: Implications of Memory, Structure and Scent for Information Retrieval," In Proceedings of CHI 1998 Conference on Human Factors in Computing Systems, April 18-23, 1998, pp. 25-32.

Lynch, P.J. Yale University C/AIM $W W W$ Style Guide. URL: http://info.med.yale.edu/caim/StyleManual-Top.HTML, 1995.

Modjeska, D. Navigation in Electronic Worlds: A Research Review. Technical Report, Computer Systems Research Group, University of Toronto, 1997.

Morville, P. "The Age of Findability," Boxes and Arrows, 29 Apr 2002, URL: http://www.boxesandarrows.com/archives/print/002595.php, accessed on 08/08/2003.

Neo, H.K. A Wayfinding Perspective In Designing An Effective Website. M.Sc. Thesis, National University of Singapore, 2003.

Neo, H.K., Tan, G. W., and Wei, K. K. "The Impact of Cognitive Mapping on Effective Website Design," In First Annual Proceedings of Special Interest Group Human Computer Interaction (SIGHCI) workshop, Barcelona, Spain, Dec 14, 2002.

Nielsen, J. Designing Web Usability: The Practice of Simplicity, Indianapolis, IN: New Riders, 2000.

Nielsen, J., and Tahir, M. Homepage Usability: 50 Websites Deconstructed, Indianapolis, IN: New Riders, 2002.

NUA. "Badly designed websites keep surfers away," TechNews.com, 2002, URL: http://www.nua.ie/surveys/index.cgi?f=VS\&art $\mathrm{id}=905357977 \&$ rel=true, accessed on $02 / 05 / 2003$.

Passini, R. Wayfinding in Architecture, New York, Van Nostrand Reinhold, 1984.

Thorndyke, P.W., and Hayes-Roth, B. "Differences in Spatial Knowledge Acquired from Maps and Navigation," In Cognitive Psychology. New York: Academic Press, 1982.

Zhang, P., Small, R.V., Dran, G.V., and Barcellos, S. "Websites that Satisfy Users: A Theoretical Framework for Web User Interface Design and Evaluation," Thirty-second Hawaii International Conference on System Sciences (HICSS), Maui, Hawaii, Jan 5-8, 1999.

Zhang, P., Dran, G.V., Blake, P., and Pipithsuksunt, V. "A Comparison of the Most Important Website Features in Different Domains: An Empirical Study of User Perceptions," Sixth Americas Conference on Information Systems (AMCIS), Long Beach, California, Aug 1013, 2000, pp. 1367-1372. 